\section{CCN posters}

\section{PRODUCTION OF A C-TERMINAL FRAGMENT OF CTGF IN ESCHERICHIA COLI THAT MIMICS ACTIVITY OF THE INTACT CTGF MOLECULE}

D.K. Ball, D.R. Brigstock. Department of Surgery, Children's Hospital, Columbus, Ohio, USA

The primary translational product of human connective tissue growth factor (CTGF) is predicted to comprise 349 residues, which, after cleavage of the signal peptide, is expected to produce a protein of 323 residues with a $M$ of 38000 . Lower mass forms of CTGF comprising modules 3 and 4 or module 4 alone appear to exhibit some of the activities of the full-length CTGF protein, suggesting that functional domains are present in the C-terminal region of CTGF. To facilitate studies of the lowest mass form of CTGF identified to date, we have used an E coli expression system to produce a $10 \mathrm{kDa}$ CTGF protein that comprises essentially module 4 and corresponds to residues 247-349 of human CTGF

The cDNA encoding $10 \mathrm{kDa}$ CTGF (commencing at Glu247) was cloned into the pMAL-c2 vector (New England Biolabs) and the construct was transformed into $E$ coli strain BL21. Escherichia coli carrying the cDNA for the maltose-binding protein-CTGF fusion protein were induced with isopropylthiogalactoside (IPTG) for one hour and then mechanically lysed using a French press. The cell extract was passed through an amylose resin and the bound fusion was eluted with $10 \mathrm{mM}$ maltose. This protein was digested with factor $\mathrm{Xa}$ and the cleavage products further purified by heparin-affinity chromatography and reverse-phase high performance liquid chromatography. Recombinant $10 \mathrm{kDa}$ CTGF demonstrated comparable immunoreactive and heparin-binding properties to native CTGF and promoted adhesion of several cell types including fibroblasts, endothelial cells, and epithelial cells. For each cell type tested, CTGFmediated cell adhesion was heparin dependent and was ablated by prior treatment of the CTGF with reducing agents.

In conclusion, recombinant $10 \mathrm{kDa}$ CTGF produced in $E$ coli appears to mimic the biological activity and heparin-binding properties of native CTGF. The intrachain disulfide bridges within $10 \mathrm{kDa}$ CTGF appear to be essential for promoting cell adhesion. This system is a viable source of truncated CTGF with which to perform structurefunction studies.

\section{O2 ANALYSIS OF GENE EXPRESSION IN OSTEOBLASTIC CELLS STIMULATED BY CONNECTIVE TISSUE GROWTH FACTOR HYPERTROPHIC CHONDROCYTE-SPECIFIC GENE PRODUCT 24 (CTGF/HCS24)}

H. Doi ${ }^{1,2}$, E. Nakata ${ }^{1,2}$, T. Nakanishi', K. Nishida ${ }^{2}, H$. Inoue $^{2}, M$. Takigawa'. 'Department of Biochemistry and Molecular Dentistry, and ${ }^{2}$ Department of Orthopaedic Surgery, Okayama University Graduate School of Medicine and Dentistry, Okayama, Japan

Connective tissue growth factor (CTGF/Hcs24) belongs to the CCN family, which is believed to regulate many biological processes. We reported that recombinant CTGF/Hcs24 (rCTGF/Hcs24) promoted the proliferation and differentiation of osteoblastic cells (Saos2) in culture and increased the expression of collagen type I, alkaline phosphatase, osteopontin, and osteocalcin. To investigate the effect of rCTGF/Hcs24 on gene expression levels in Saos2 in more detail, we used DNA microarrays. Saos2 cells cultured in DMEM containing $10 \%$ fetal bovine serum (FBS) for 48 hours were then incubated in DMEM containing $0.5 \%$ FBS for 12 hours. Then, the medium was changed to DMEM containing $0.5 \%$ FBS or DMEM containing $0.5 \%$ $\mathrm{FBS}$ and $50 \mathrm{ng} \mathrm{rCTGF} / \mathrm{Hcs} 24$. DNA microarrays containing 1091 human genes were used to compare mRNA values between cultures treated with or without rCTGF/Hcs24 at six, 12, and 24 hours after the addition. At each time point, we identified genes whose expression was altered by $\mathrm{rCTGF} / \mathrm{Hcs} 24$. After 6 hours of treatment with rCTGF/Hcs24, two genes were upregulated and eight genes were downregulated. After 12 hours, 22 genes were upregulated and 38 genes were downregulated. After 24 hours of incubation, 14 genes were upregulated and seven genes were downregulated. These results show that DNA microarrays are a useful means of identifying the expression patterns of mRNA involved in the cellular response to rCTGF/Hcs24.

\section{O3 A CENTRAL ROLE FOR CTGF IN ECM REMODELLING DURING FOLLICULOGENESIS?}

A.C. Bradshaw, M. Rae, C.R. Harlow, S.G. Hillier. Dept of Reproductive and Developmental Sciences, University of Edinburgh, Chancellor's Building, 49 Little France Crescent, Edinburgh, Scotland, UK

Ovarian folliculogenesis-the cyclical growth, development, and atresia of ovarian follicles-is characterised by extensive remodelling of the extracellular matrix (ECM) to enable follicle expansion. Matrix metalloproteinases (MMPs) are key factors in ECM breakdown during folliculogenesis, but little is known of how ECM deposition is regulated in the ovary.

We have recently identified CTGF and lysyl oxidase (LOX) as sequentially expressed, transforming growth factor $\beta$ (TGF- $\beta$ ) regulated genes in rat ovarian granulosa cells. We now present in situ hybridisation computer-assisted image analysis on the developmental expression of CTGF and P450 aromatase during folliculogenesis, and CTGF regulated mRNA expression of LOX, procollagen-C-proteinase (PCP; enhances LOX activity and procollagen processing), $P C P$ enhancer (PCPE; potentiates PCP activity), CTGF itself, and CTGF regulated MMP activity, in 48 hour cultures of granulosa cells from DES- (stimulates preantral/early antral follicular development) or PMSG-treated 21 day rats.

In ovaries from DES-treated rats, there was a significant positive correlation between follicle size and CTGF mRNA expression. The relative intensity of expression in preantral follicles was $2.95 \pm 0.55$ $(60-120 \mu \mathrm{m}$ diameter; $\mathrm{n}=11)$ and $4.70 \pm 0.36(121-165 \mu \mathrm{m}$ diameter; $\mathrm{n}=34)$ and in antral follicles $11.77 \pm 2.62(166-275 \mu \mathrm{m}$ diameter; $\mathrm{n}=9)$ and $17.37 \pm 3.9(276-500 \mu \mathrm{m}$ diameter; $\mathrm{n}=8)$. The corresponding relative $\mathrm{P} 450$ aromatase mRNA expression was 0.43 $\pm 0.23(n=14), 0.74+032(n=7), 0.98+0.21(n=9)$, and 1.77 $\pm 0.25(n=7)$, respectively. Whereas there was a further fourfold increase in $\mathrm{P} 450$ aromatase to $6.82 \pm 0.63(\mathrm{n}=9)$ in preovulatory follicles 48 hours after PMSG, in contrast, CTGF mRNA fell by $95 \%$ to $0.83 \pm 0.23(n=8)$. In vitro, LOX, PCP, PCPE, and CTGF mRNA were all enhanced dose-dependently up to two to sixfold by recombinant human CTGF $(1-30 \mathrm{ng} / \mathrm{ml})$. MMP-9 was unaffected by CTGF, whereas MMP-2 activity was dose-dependently enhanced up to fivefold by $100 \mathrm{ng} / \mathrm{ml}$ CTGF.

These results confirm an increase in CTGF up to the antral stage, declining rapidly as oestrogen biosynthesis takes off, and suggest that CTGF may mediate TGF- $\beta$ stimulation of LOX. Furthermore, CTGF may potentiate LOX activity and collagen deposition by enhancing PCP and PCPE expression in the ovary. Importantly, CTGF raises MMP-2 activity in granulosa cells, providing a mechanism whereby CTGF may also be involved in ECM degradation. We therefore propose that CTGF may play a pivotal role in tissue remodelling in the ovary.

We thank Fibrogen Inc, San Francisco, California, USA for providing the recombinant human CTGF.

\section{THE CCN-5 CHICK HOMOLOGUE: EXPRESSION ANALYSIS AND FUNCTIONAL STUDIES}

B.E de Oliveira, J.A. Jones, J. Castellot. Program in Cell, Molecular and Developmental Biology, Tufts Sackler School of Graduate Biomedical Sciences, Boston, Massachusetts, USA

CCN5 is a heparin-induced gene that shares structural homology with the CCN family of secreted, cysteine rich proteins involved in matrix synthesis, growth regulation and cell motility. CCN5 was identified in our laboratory by subtractive hybridization. Although CCN5 expression in the adult mouse and rat has been studied, little is known about embryonic expression of the molecule in any species. Here, we report the spatial and temporal expression of CCN5 in the embryonic development of the chick and mouse, using in situ hybridization. For embryonic expression in the chick, we first identified the chicken homologue of the CCN5 gene, which was $76 \%$ identical to the human gene and $75 \%$ identical to the mouse gene. We then developed and characterized a RCAS retrovirus for the delivery of chick CCN5. 
Overexpression studies were carried out by infecting the chorioallantoic membrane, or by microinjecting the retro-CCN5 constructs into the embryonic chick limb, with particular emphasis on analyzing the functional role of CCN5 in the vasculature of the developing limb. We have also characterized an anti-CCN5 antibody that permits localization of CCN5 in rodents, and are in the process of using this antibody to examine the spatial and temporal expression pattern in mouse embryos using immunohistochemical analysis.

\section{THE NOV (CCN3) GENE IS NOT A COMMON TARGET IN MAV1(N)-INDUCED NEPHROBLASTOMAS}

C.L. Li, R. Zoorob', C. Auffray', P. Coullin², B. Perbal. Laboratoire d'Oncologie Virale et Moléculaire, UFR de Biochimie, Université Paris 7-D. Diderot, Paris; 'IRSC and ${ }^{2}$ GGR Villejuif, France

By screening $\lambda$ libraries of MAVI $(N)$ induced nephroblastomas we discovered that in one tumor a MAV proviral genome was integrated within the gene that we designated nov (for nephroblastoma overexpressed) because its expression is raised in all the nephroblastomas that we have analyzed. However, the downstream promotion of nov was detected in a single tumor in which the chimeric mRNA initiated in the MAV long terminal repeat (LTR) encoded a truncated protein that had oncogenic properties. To establish whether the high amounts of nov mRNAs detected in avian tumors resulted from the stimulation of transcription directed by LTR enhancers localized in the vicinity of the nov gene, we mapped the MAV sequences in the DNA of three tumors, representing various stages of tumor development. Because MAV-induced nephroblastoma is a unique paradigm for Wilms's tumor, we also reasoned that the characterization of MAV integration sites might prove fruitful in deciphering the molecular basis of this frequent pediatric cancer. Hybridization of the MAVI(N) U3 specific probe to tumor DNA fragments separated by field inversion gel electrophoresis indicated that MAVI was integrated in a limited number of sites in the genome of tumor cells. The relatively small size of the DNA fragments cloned in the $\lambda$ libraries that we originally used did not permit us to detect MAV proviral genomes at larger distances. To establish a physical map of the various MAV integration sites a series of 23 junction fragments derived from the three independent tumors were used as probes for screening BAC (bacterial artificial chromosomes) libraries. A total of 78 BACs containing MAV integration sites were isolated and analyzed. BAC fragments containing MAV sequences have been characterized and used to perform fluorescent in situ hybridization on the chicken chromosomes. The results we obtained enabled us to localize the chicken nov gene to chromosome 2q34-36 and to show that MAV integration sites in the tumor DNA are restricted to a few chromosomes. The results obtained indicated that the nov gene is not a preferential target for MAV integration in nephroblastomas. Other genes whose functions are relevant to tumor development have been identified as potential integration sites. These observations raise interesting questions as to the mechanisms responsible for the increased levels of NOV transcripts detected in the MAV-induced nephroblastomas and the relation that might exist between the different genes whose expression is induced in these tumors.

\section{A ROLE FOR CCN3 (NOV) IN CALCIUM SIGNALING}

A. Lombet, C.L. Li' V. Martinez' B. He ${ }^{2}$, B. Perbal'. Unité Inserm 339, Hôpital Saint-Antoine, Paris, France; 'Laboratoire d'Oncologie Virale et Moléculaire (LOVM), Université Paris 7-D. Diderot, Paris, France; ${ }^{2}$ Department of Microbiology and Immunology, University of Illinois, Chicago, USA

In animal and humans increased expression of CCN3 (NOV) has been detected in various tissues where calcium is a key regulator. Because the multimodular structure of the $\mathrm{CCN}$ proteins is strongly suggestive of these cell growth regulators being metalloproteins, we have searched for a possible role of CCN3 in ion flux and transport during development, control of cell proliferation, and differentiation and physiology.

To establish whether the CCN3 protein affects calcium ion fluxes, we used a dynamic imaging microscopy system allowing fluorometric measurement of intracellular calcium concentrations.

The use of glutathione-S-transferase fusion proteins allowed us to establish that the addition of CCN3 to G59 glioblastoma and SK-N-SH neuroblastoma cells produced a pronounced transient increase of intracellular calcium, originating from both the entry of extracellular calcium and the mobilization of intracellular stores.

The increased intracellular calcium concentrations induced by CCN3 involve different processes, among which voltage-independent calcium channels might be of considerable importance in regulating the calcium flux associated with cell growth control, motility, and spreading.

Our observations assign a biological function to the $\mathrm{CCN} 3$ protein for the first time and point out a broader role for the CCN proteins in calcium ion signaling.

07 FUNCTIONAL ANALYSIS OF CONNECTIVE TISSUE GROWTH FACTOR (CTGF/CCN2) IN CALCIFYING TISSUES USING TRANSGENIC MICE AND ITS FUNCTIONAL CORRELATION WITH BONE-FORMING TRANSCRIPTION FACTOR CBFA1

T. Nakanishi, T. Yamaai', K. Nawachi, G. Yoshimichi, T. Eguchi, T. Komori $^{2}$, T. Sugimoto', M. Takigawa. Department of Biochemistry and Molecular Dentistry and 'Oral Functional Anatomy, Okayama University Graduate School of Medicine and Dentistry, Okayama, Japan; ${ }^{2}$ Department of Medicine III, Osaka University Medical School, Suita, Japan

Connective tissue growth factor (CTGF/CCN2) is produced by chondrocytes, vascular endothelial cells, and transforming growth factor $\beta$ (TGF- $\beta$ )-stimulated fibroblasts. We previously reported that CTGF was isolated from a chondrosarcoma-derived cell line and normally expressed in cartilage tissues, especially in hypertrophic chondrocytes. We also showed that CTGF stimulated both the proliferation and differentiation of chondrocytes in vitro. Therefore, CTGF is thought to be one of the most important regulators of endochondral ossification in vivo. Here, we show the function of CTGF in vivo using transgenic mice that overproduce CTGF/Hcs 24 under the control of the mouse type $\mathrm{XI}$ collagen promoter and also show its functional correlation with bone-forming transcription factor Cbfa $\mathrm{l}$.

These transgenic mice seemed to develop normally and their embryonic and neonatal growth was also normal. However, they showed dwarfism within a few months of birth and $x$ ray analysis revealed that their bone density was decreased compared with normal mice. The femur in the hind limbs in particular showed an apparent low density. These results indicated that overexpression of CTGF/Hcs24 affects certain steps of endochondral ossification. Boneforming transcription factor $\mathrm{Cbfal}$, which has already been shown to promote chondrocyte differentiation, was expressed from the zone of hypertrophic chondrocytes to calcifying cartilage in the ribs of normal mouse neonates. Surprisingly, the expression of ctgf, which was detected in the zone of hypertrophy, and provisional calcification, in which ossification proceeds toward the epiphysis during the skeletal development of the mouse embryo, was completely abolished in ribs, phalanges, and vertebrates of cbfa 1 -null embryos, and ctgf-transgenic mice rescued the cartilage differentiation of cbfal-null mice by promoting chondrocyte hypertrophy.

These results indicate the important roles of CTGF in the embryonic development of calcifying tissues in the mouse, and also suggest a functional correlation of CTGF with Cbfal during chondrocyte maturation.

\section{8 rAAV-MEDIATED CTGF GENE OVEREXPRESSION IN SKELETAL MUSCLE IN VIVO IS ASSOCIATED WITH MUSCLE FIBER ATROPHY}

A. Wilson Rachfal, K. Reed Clark', M. Luquette ${ }^{2}$ D.R. Brigstock. Departments of Surgery, Pediatrics' and Pathology', Children's Hospital, Columbus, Ohio, USA

In vitro studies have shown that connective tissue growth factor (CTGF) stimulates a broad spectrum of cellular activities including mitosis, migration, angiogenesis, apoptosis, and the production of extracellular matrix proteins. Although considerable attention has been focused on the potential role of CTGF in fibrotic disease, few studies have directly investigated the biological functions of CTGF in vivo. To address this question, we have developed a recombinant adenoassociated virus (rAAV) carrying CTGF cDNA downstream of the cytomegalovirus (CMV) promoter for the transduction of tissues in vivo. Major advantages of rAAV over other viral delivery systems are that it is non-pathogenic, non-immunogenic, and infective for non-dividing cells.

A recombinant virus encoding full length CTGF was engineered using a plasmid containing viral long terminal repeats, rep, and cap genes, in addition to the CMV promoter and the BHG polyadenylation signal. Virus was generated by stably transfecting this construct into Hela cells and treating them with adenovirus serotype 5, resulting in a productive infection yielding rAAV CTGF virus. After purification of the 
recombinant virus, $5^{\text {ell }}$ CTGF viral particles were injected into the quadricep of 3-4 week old mice. Skeletal muscle is a well-established target for AAV-mediated gene transduction. Eight weeks after injection, viral integration was tested by the polymerase chain reaction (PCR), mRNA production was tested by reverse transcription $\mathrm{PCR}$, and protein production was tested by immunohistochemistry.

Comparison of infected muscle with non-infected muscle showed viral persistence and RNA production in infected muscle. Histological examination showed groups of abnormal fibers, many of which were atrophic. Both the atrophic fibers and adjacent non-atrophic fibers show interfibrillar inclusions composed of a granular substance, which was determined to be CTGF by immunostaining.

These data show that rAAV is a viable means of delivering the CTGF gene in vivo and of studying the effects of its overexpression. When expressed in the quadriceps, CTGF was overproduced in distinct muscle cells and accumulated at high intracellular levels, a phenomenon that was associated with muscle fiber atrophy. These data suggest that skeletal muscle is a target for CTGF action and that high intracellular concentrations of CTGF are pathogenic.

\section{CCN FAMILY MEMBER EXPRESSION IN CARTILAGE NEOPLASIA}

A-T. Le, C. Yu, H. Yeger, B. Perbal', B.A. Alman. Program in Developmental Biology of the Hospital for Sick Children, Toronto, Ontario, Canada; 'UFR de Biochimie, Université Paris 7-D. Diderot, Paris, France

The CCN family (cysteine-rich CYR61, connective tissue growth factor (CTGF), and nephroblastoma overexpressed gene (NOV)) of genes encode structurally related proteins, many of which play a role in cell differentiation. As a group, cartilage lesions are the most common tumours of bone. They range from benign lesions, such as enchondromas (which arise from growth plate chondrocytes that fail to undergo terminal differentiation) to malignant chondrosarcomas. Because several CCN family members are expressed in cartilage development, the growth plate, and in sarcomas, they may be differentially regulated in different grades of cartilage tumours. This has already been demonstrated for CTGF. We studied three cases of enchondromas, seven low grade chondrosarcomas, four medium grade chondrosarcomas, and four high grade chondrosarcomas. Using real-time polymerase chain reaction, we measured levels of expression of NOV (CCN3), CYR61, CTGF, and WISP1, normalised to the glutaraldehyde-3-phosphate dehydrogenase (GAPDH) gene. We found that there was decreased expression in higher-grade chondrosarcomas compared with enchondromas in all four genes. When the levels of expression of all four genes was averaged, there was a clear difference between the expression of the enchondromas and low-grade chondrosarcomas, and between the different grades of chondrosarcomas. Because the identification of the grade of cartilage neoplasia can sometimes be difficult using routine histology, the expression of CCN family members might be a useful adjunct in determining tumour grade.

\section{CONNECTIVE TISSUE GROWTH FACTOR IS NECESSARY FOR GENES REQUIRED FOR ANGIOGENESIS}

S. Ivkovic ${ }^{1 *}$, B.S. Yoon ${ }^{2 *}$, K.M. Lyons ${ }^{1,2,3}$. 'Departments of Orthopaedic Surgery, ${ }^{2}$ Molecular, Cell and Developmental Biology, and ${ }^{3}$ Biological Chemistry, University of California, Los Angeles, 90095, USA

We have shown that connective tissue growth factor (CTGF), a secreted factor, regulates the composition of extracellular matrix (ECM) components during the development of endochondral bones. As a consequence, developmental malformations of bones, ribs, vertebral bodies, and skull were found in Ctgf deficient mice. At the histological level, we saw an expansion of the hypertrophic zones in the radius/ulna and tibia/fibula, which suggested a possible impairment in angiogenesis, because expansion of the hypertrophic zone has been associated in several studies with impaired invasion of the hypertrophic ECM by blood vessels. Our analysis of expression of genes thought to be involved in growth plate angiogenesis showed that several are downregulated in Ctgf deficient mice. For example, immunostaining of the radius and una show decreased concentrations of vascular endothelial growth factor (VEGF) and matrix metalloproteinase 13 (MMP13) in the hypertrophic zones of mutant mice in comparison to wild-type littermates. Further analysis by semiquantitative reverse transcription polymerase chain reaction of cartilage extracts from embryonic day 17.5 mice showed that there is a decrease in Vegf mRNA values in the mutant, indicating that Ctgf regulates the transcription of Vegf in the hypertrophic zone. In contrast, there was no difference in transcriptional levels of $\mathrm{Mmp} 13$ between the mutant and wild-type littermates. To examine other possible modes of regulation, we performed co-immunoprecipitation with CTGF and MMP13. We found that CTGF can bind to MMP13, indicating a possible role for CTGF in the post-translational regulation of MMP13 stability.

Therefore, our results show that CTGF is necessary for the expression of genes required for angiogenesis during endochondral ossification of long bones.

*These authors contributed equally to the work.

\section{PROTEIN KINASE C MEDIATES REGULATION OF CHONDROCYTE DIFFERENTIATION AND} PROLIFERATION OF CTGF/HCS24 VIA MAPK SIGNALING

G. Yosimichi' ${ }^{13}$, S. Kubota', T. Nishida', S. Kondo', K. Nakao', T. TakanoYamamoto $^{2}$, M. Takigawa', ${ }^{1,3}$. 'Department of Biochemistry and Molecular Dentistry, ${ }^{2}$ Department of Orthodontics, Okayama University Graduate School of Medicine and Dentistry, Okayama, Japan; ${ }^{3}$ Biodental Research Center, Okayama University Dental School, Okayama, Japan

Connective tissue growth factor/hypertrophic chondrocyte specific gene product 24 (CTGF/Hcs24) promotes the proliferation and differentiation of chondrocytes in culture. Previously, we reported that extracellular signal-regulated protein kinase (ERK) mediates the CTGF/Hcs24-induced proliferation of chondrocytes, and p38 mitogen activated protein kinase (MAPK) mediates the CTGF/Hcs24induced differentiation of chondrocytes (G Yosimichi et al. Eur J Biochem 2001;268:6058-65). Here, we report the cell biological effects of a protein kinase C (PKC) inhibitor (Calphostin C), PKA inhibitor $(\mathrm{H}-89)$, and phosphatidylinositol 3 kinase (PI3K) inhibitor (wortomannin) on HCS-2/8 cells stimulated by CTGF/Hcs24. CTGF/ Hcs24-induced enhancement of proteoglycan synthesis was inhibited by PKC and 38 MAPK inhibitors in a dose dependent manner in HCS-2/8 cells. In agreement, the PKC inhibitor, but not the PKA and PI3K inhibitors, suppressed the phosphorylation of p38 MAPK induced by CTGF/Hcs24. In addition, proliferation of HCS-2/8 cells was suppressed drastically by the PKC inhibitor. Moreover, CTGF/Hcs24 increased the activation of PKCa and PKCe, but not PKCz. In rabbit growth cartilage cells, alkaline phosphatase (ALP) activity, accumulation of proteoglycan, and DNA synthesis, which were all stimulated by CTGF/Hcs24, were inhibited by the PKC inhibitor, whereas the PI3K inhibitor suppressed only ALP activity. In addition, the PKA inhibitor suppressed the phosphorylation of ERK by CTGF/Hcs24, and the PKA and PI3K inhibitors suppressed the phosphorylation of MEK 1. Moreover, CTGF/Hcs24 induced PKB phosphorylation 10 minutes after stimulation, but did not affect the cAMP concentration. These results suggest that PKC is a major mediator of chondrocyte differentiation and proliferation stimulated by CTGF/ Hcs24, whereas PKB, PI3K and PKA play only minor roles.[AQ:2]

\section{PROANGIOGENIC ACTIVITIES OF CYR61 (CCN1) MEDIATED THROUGH INTEGRINS $\alpha_{4} \beta_{3}$ AND $\alpha_{6} \beta_{1}$ IN HUMAN UMBILICAL VEIN ENDOTHELIAL CELLS}

S-J. Leu, S.C-T. Lam', L.F. Lau. Departments of Molecular Genetics and Pharmacology', University of Illinois College of Medicine, 900 South Ashland Avenue, Chicago, IL 60607-7170, USA

CYR61 (CCN1) is an extracellular matrix-associated protein of the CCN family, which also includes CTGF (CCN2), NOV (CCN3), WISP-1 (CCN4), WISP-2 (CCN5), and WISP-3 (CCN6). Purified CYR61 induces neovascularization in corneal implants and Cyr61-null mice suffer embryonic death as a result of vascular defects, thus establishing that CYR61 is an important regulator of angiogenesis. Aberrant expression of CYR61 is associated with breast cancer wound healing, and vascular diseases such as atherosclerosis and restenosis. In culture, CYR61 functions through integrin-mediated pathways to promote cell adhesion, migration, and proliferation. Here, we show that CYR61 can also promote cell survival and tubule formation in human umbilical vein endothelial cells (HUVECs). Furthermore, we have dissected the integrin receptor requirements of CYR61 with respect to its proangiogenic activities. Thus, CYR61-induced cell adhesion and tubule formation occur through interaction with integrin $\alpha \beta_{1}$ in early passage endothelial cells where integrins have not been activated. In contrast, in endothelial cells where integrins are activated by phorbol ester or vascular endothelial growth factor, CYR61promoted cell adhesion, migration, survival, growth factor-induced mitogenesis, and endothelial tubule formation are all mediated 
through integrin $\alpha_{v} \beta_{3}$. These data show that the proangiogenic activities of CYR61 in activated and resting endothelial cells are differentially mediated through integrins $\alpha_{v} \beta_{3}$ and $\alpha_{\beta} \beta_{1}$, respectively. These findings help to define the mechanisms by which CYR61 acts as an angiogenic regulator, provide a molecular interpretation for the loss of vascular integrity and increased apoptosis of vascular cells in Cyr61-null mice, and underscore the importance of CYR61 in the development and homeostasis of the vascular system.

\section{EXPRESSION OF CONNECTIVE TISSUE GROWTH FACTOR/HYPERTROPHIC CHONDROCYTE-SPECIFIC GENE PRODUCT 24 (CTGF/HCS24) DURING FRACTURE} HEALING

E. Nakata ${ }^{1,2}$, T. Nakanishi', H. Doi ${ }^{1,2}$, H. Inoue ${ }^{2}$, M. Takigawa'. 'Department of Biochemistry and Molecular Dentistry, and 'Department of Orthopaedic Surgery, Okayama University Graduate School of Medicine and Dentistry, Okayama, Japan

The localization and expression of connective tissue growth factor/hypertrophic chondrocyte-specific gene product 24 (CTGF/ Hcs24) during fracture healing in mouse ribs were investigated. To prepare samples for immunostaining and in situ hybridization, the ribs from a total of 50 mice were fixed at $0,2,8,14$, and 20 days. In situ hybridization demonstrated that CTGF/Hcs 24 mRNA was highly expressed, especially in hypertrophic chondrocytes and proliferating chondrocytes in the regions of regenerating cartilage on days 8 and 14 after the fracture. CTGF/Hcs24 mRNA was also expressed in proliferating periosteal cells in the vicinity of the fracture sites on days 2 and 8 , and in cells in fibrous tissue around the callus on day 8 . Northern blot analysis showed that the expression of CTGF/Hcs 24 mRNA was 3.9 times higher on day 2 of fracture healing than that seen on day 0 . On day 8 , it reached a peak of 8.6 times higher than that seen on day 0 . Thereafter, it declined to a lower level. Immunostaining showed that CTGF/Hcs24 was localized in hypertrophic chondrocytes and proliferating chondrocytes in the regions of regenerating cartilage, and in active osteoblasts in the regions of intramembranous ossification. Although CTGF/Hcs24 was abundant in the proliferating and differentiating cells (on days 8 and 14), the immunostaining decreased as the cells differentiated to form bone (on day 20). CTGF/Hcs24 was also detected in cells in fibrous tissue, vascular endothelial cells in the callus, and periosteal cells around the fracture sites. These results suggest that CTGF/Hcs24 is an important regulator in chondrogenesis, osteogenesis, and angiogenesis in vivo, and promotes intramembranous ossification and endochondral ossification in the processes of fracture healing.

New JCP online submission and review system

We are pleased to inform authors and reviewers of the new online submission and review system at JCP. Developed by HighWire Press (CA, USA), Bench Press is a fully integrated electronic system that utilises the web to allow rapid and efficient submission of manuscripts. It also allows the peer review process to be conducted entirely online. We are one of the first journals in the BM Special Journals group to go online in this way. The aim, apart from saving trees, is to speed up the often frustratingly slow process (for both authors and editors) from submission to publication. Many reviewers might appreciate this too. Authors may submit their manuscript in any standard word processing software. Acceptable standard graphic formats include: jpeg, tiff, gif, and eps. The text and graphic files are automatically converted to PDF for ease of distribution and reviewing purposes. Authors are asked to approve their submission before it formally enters the reviewing process. On approval by the authors, the submission is passed to the editor and/or reviewers via the web. All transactions are secure.

To access the system click on "SUBMIT YOUR MANUSCRIPT HERE" on the JCP homepage: HYPERLINK http://www.jclinpath.com, or you can access Bench Press directly at HYPERLINK http://submit-jcp.bmjijournals.com.

We are very excited with this new development and would encourage authors and reviewers to use the online system whenever possible. As editors, we will use it all the time, the up side being lack of need to travel to the editorial office to deal with papers, the down side having no more excuses to postpone decisions on papers because we are "at a meeting"!

The system is very easy to use and should be a big improvement on the current peer review process. Full instructions can be found on Bench Press http://submit-jcp.bmijournals.com and JCP online at http://www.jclinpath.com. Please contact Natalie Davies, Project Manager, HYPERLINK mailto:ndavies@bmjgroup.com for any further information. 